\title{
Digital archiving project of paper maps collected for the International Map Exhibition 1980 in Tokyo
}

\author{
Hideki KAJI ${ }^{\text {a, }}$, Ken'ichi TSURUOKA ${ }^{\text {a }}$, Ruochen SI ${ }^{\text {a }}$, Min LU ${ }^{\text {b }}$, Masatoshi ARIKAWA ${ }^{\text {a }, ~}$, \\ Takashi OGUCHI ${ }^{\mathrm{a}}$ \\ ${ }^{a}$ Center for Spatial Information Science, The University of Tokyo, Japan. kaji@csis.u-tokyo.ac.jp, tsuruoka@csis.u-tokyo.ac.jp, \\ si@csis.u-tokyo.ac.jp,arikawa@ie.akita-u.ac.jp,oguchi@csis.u-tokyo.ac.jp \\ ${ }^{b}$ Faculty of Arts and Science, Kyushu University, Japan. lu@artsci.kyushu-u.ac.jp \\ ${ }^{c}$ Graduate School of Engineering Science, Akita University, Japan. \\ * Corresponding author
}

Keywords: Digital Archive, Old Paper Maps, Web Applications

Abstract:

The Kashiwa Library (KL), The University of Tokyo, holds a collection of old paper maps over the world, about a half of which were originally collected for the International Map Exhibition 1980 in Tokyo. The collection has 3,200 maps published in the 1970s and 1980s, and 1,260 of them were displayed at the exhibition. The map collection is important because it represents the cartography at the emerging era of new technologies and techniques such as satellite remote sensing, computers and GIS for map production (Arikawa et al., 2016). These maps were donated from the Japan Cartographers Association in March 2016, after their collection and storage by the association since the exhibition. In the Japanese fiscal year 2017, the Center for Spatial Information Science (CSIS), The University of Tokyo, and KL started a cooperative research project to produce a digital archive of this map collection, with support from the University of Tokyo Academic Archives Project that facilitates digital archiving of academic materials owned by various units at the university. This presentation explains the procedure of making our digital archive "Kashiwanoha Paper Maps Digital Archive". "Kashiwanoha" is the address of the Kashiwa Campus of The University of Tokyo where KL and CSIS are located, and it literally means "oak leaf".

There are three steps for opening the archive to the public: (1) conversion of analogue paper maps into digital ones, (2) developing a digital archive system for storing and browsing the digital maps, and (3) license applications for publishing the maps on the web.

(1) Conversion of paper maps into digital ones: We tried to make map images as detailed as possible for enhancing the value of the archive and presenting high-resolution images to users. The method of digital conversion is simple; we used a large size scanner for sheets up to A0 size to obtain 600 dpi high-resolution images of the paper maps. However, a part of the collection could not be scanned because some maps have been damaged or are thicker than the capacity of the scanner. We tried to use photo stitching methods with handy cameras for conversion of these maps.

(2) Developing a digital archive system: To make our digital archive open to public, we developed a web application for users to search map data and browse high-resolution digital map images with a web browser. The front-end user interfaces of our system are coded by JavaScript and HTML5, and the back-end server scripts are coded by Perl. The web interface displays boundary boxes of maps on the tiles of OpenStreetMap@ (https://www.openstreetmap.org/). This function allows users to understand geographical locations of the images (Figure 1). In the map image view, users can browse each map image in detail with using a tracking and scaling function, and can read 16 attribute data such as Title, Scale, Topic, Author, and Description (Figure 2).

(3) License applications: We have been making license applications for web publication to the creators or publishers of the maps. This process is taking time because the list of creators and publishers includes a lot of individuals as well as private and government organizations, and some countries that provided maps have changed their status drastically since 1980. As of December 2018, we have obtained licenses for about 220 maps from four Japanese organizations. We have been discussing related issues such as the way to publish the digital map images and their fair use policy.

In November 2018, we opened our digital archive system to public (URL: https://maparchive.csis.u-tokyo.ac.jp/browse/). As of January 2019, anyone with web access can see 41 map images and metadata using our system. We will keep developing the system and making license applications to expand the contents to enhance the value of this digital archive. Especially, we need to implement functions to produce metadata features efficiently. Currently, making metadata requires a great effort because we need to read information from each map manually, if we do not have any other document about it. A possible better way to make metadata is to use the crowd sourcing method by ordinary users like the case of OpenStreetMap. Another possible way is metadata extraction using an appropriate image processing method. Either way can be adapted to not only making metadata but also vectorizing maps, which will make our system and the 
digital archive more useful and valuable. We will also attempt to publish the digital archive system itself as an open source software. We expect such an attempt will lead to effective utilization of hoarded paper maps all around the world.

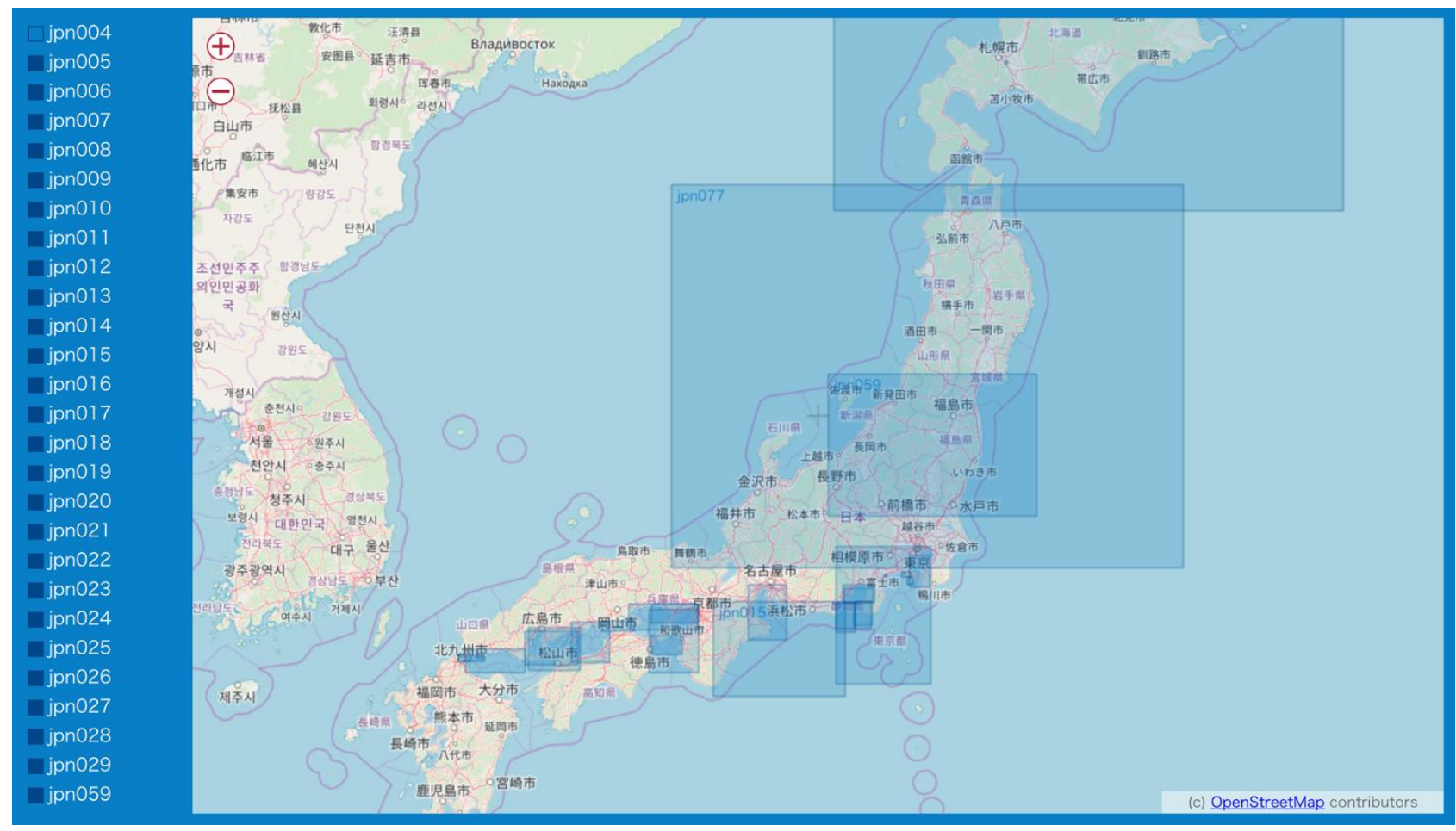

Figure 1. Main view of our web system showing a list of map names on the left side and blue colored boundary boxes of viewable maps on OpenStreetMap.

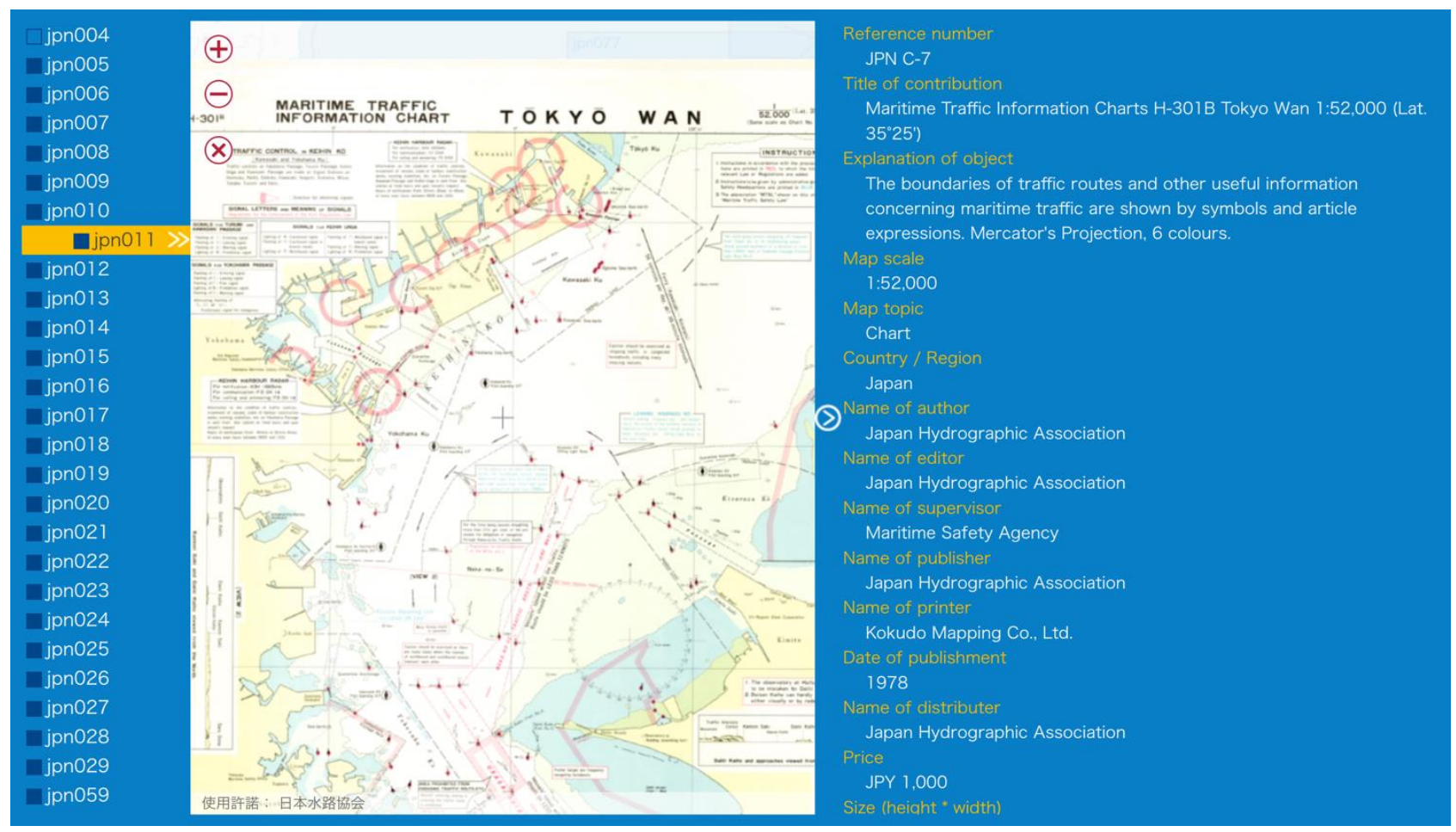

Figure 2. Map image view showing a digital map image and metadata of a selected map.

\section{Reference:}

Masatoshi ARIKAWA, Kenzo IMAI, Hiroshi OTA, Junko SUSUKI, Takashi MORITA, 2016. IMY Memorial Cooperative Workshop "Appreciate and learn maps over the world, and discuss map culture", Japan Cartographers Association, Journal MAP, 54(2) (In Japanese). 